\title{
Model and implementation of pseudorange-bias-free linear channel
}

\author{
Yanhong Kou ${ }^{1}$ (I) Huiwen $\mathrm{Wu}^{1}$
}

Received: 10 August 2020 / Accepted: 15 January 2021 / Published online: 10 February 2021

(c) The Author(s) 2021

\begin{abstract}
The nonideal characteristics of the entire channel of satellite navigation signals from generation, propagation to reception will cause signal distortions, resulting in pseudorange biases. Such kind of biases cannot be eliminated by differential technologies and has become a core error source in high-accuracy applications. We study the theoretical model and implementation method of a pseudorange-bias-free linear channel. The ideal channel transfer function equation under the unbiased pseudorange requirement is first derived from the equivalent baseband model. Based on two corresponding criteria and the simulation of the influence of different amplitude- and phase-frequency responses, a digital phase compensation method based on an all-pass filter is proposed to eliminate pseudorange biases. Then the significant effects of the two phase equalizers are validated by a simulation example of the BPSK(10) signal. Finally, the real BDS3 PRN32 and PRN33 satellite B1C signals collected by a 40-m high-gain dish antenna are utilized to invert the channel transfer characteristics and processed by our software receiver. The measurement results demonstrate that the phase equalizers constructed according to either the linear phase criterion or the linear phase plus even-symmetric phase criterion can effectively reduce the pseudorange bias. The model and method provide a reference for payload and receiver optimization and are suitable for various signal structures and applications.
\end{abstract}

Keywords Pseudorange bias · Transfer function · Channel equalization · Signal distortion · Equivalent baseband model · All-pass filter

\section{Introduction}

The pseudorange measurement of a satellite navigation receiver is derived from its estimate of the code phase of the received satellite signal, which is generally obtained by the code tracking loop, alternatively called delay lock loop (DLL). The DLL discriminates the code phase error between the input signal and the ideal local replica code based on their cross-correlation function (CCF) (Ward 2017; Misra and Enge 2006). The zero-crossing point of the code loop phase discriminator curve, also known as S-curve, is also the code loop lock point. Ideally, the CCF should drop symmetrically and smoothly on both sides of the main peak, and the code loop should be locked where the code phase error is zero (Spilker 1996; Phelts 2001). However, when the signal passes through the satellite-receiver channel, various

Yanhong Kou

kouy@buaa.edu.cn

1 School of Electronics and Information Engineering, Beihang University, Beijing 100083, China imperfections of many components of the cascade channel can cause linear or nonlinear distortions of the signal arriving at the entrance of the digital receiver correlator. These distortions will lead to CCF deformations, making the code loop lock point deviate from the true code phase alignment point and thus resulting in pseudorange biases (Soellner et al. 2002; Betz 2002; He et al. 2020; Vergara et al. 2020). Since different signal modulations, satellite-receiver channel characteristics, and signal processing methods have different effects on pseudorange biases, such biases cannot be eliminated by means of differential techniques and are hard to assimilate into existing system error parameters. It has become a new error source that restricts high-accuracy/highintegrity applications and has attracted the attention of more researchers in recent years.

Pseudorange biases are of much concern to satellite navigation applications that use measurements from multiple satellites, multiple receivers, multiple signal components, multiple frequencies, or multiple systems (ICAO 2001; Arikan et al. 2008; HaKansson et al. 2017; Phelts et al. 2014; Pagot et al. 2018). For example, using the satellite-specific 
bias parameters in signal-in-space (SIS) interface control documents (ICDs) and limiting the receiver front-end bandwidth and correlator spacing cannot completely eliminate biases caused by channel imperfections because the impacts of nonideal characteristics of the receiver channel and the mechanism of its interaction with the satellite channel characteristics are neglected (Montenbruck et al. 2017; Liu et al. 2019b; Vergara et al. 2020). In addition to the group delay $\left(T_{\mathrm{GD}}\right)$ parameters broadcast in live navigation messages, some branches of international GNSS service (IGS) also release post-processing differential code bias (DCB) products to correct pseudorange biases. Traditionally, DCB is divided into satellite- and receiver-dependent parts, which are considered to be incurred by the hardware delay of the individual satellite and the individual receiver, respectively. Therefore, it is usually assumed that the satellite-dependent part is the same for different receivers, and the receiverdependent part is the same for different satellites (Wang et al. 2016; Liu et al. 2019a). A good summary of pseudorange bias issues with IGS and multi-GNSS products can be found in Montenbruck et al. (2017). It was suggested to use dedicated receiver-group-specific satellite bias values as an alternative to the present assumption of receiver-independent satellite biases. This was considered as an option in the new Bias-SINEX format (Schaer 2016). Wang et al. (2020) found that the maximum observable-specific code biases (OSB) root-mean-square (RMS) difference between different groups of receivers was more pronounced for GLONASS than for GPS and that different groups of several receiver brands that exhibit similar OSBs as well as the bias characteristics between the new signals of new GNSS constellations have to be further investigated.

The biases caused by the nonideal characteristics of the channel hardware directly leads to the degradation of key technical indicators and service performance of satellite navigation systems and their augmentation systems and derivative products. It is necessary to eliminate or mitigate their impacts from the perspective of improving analog hardware and digital signal processing design. Because it is a complex nonlinear process from nonideal channel characteristics to pseudorange biases, most existing analyses rely on simulation and test. Thöelert et al. (2014) and Vergara et al. (2016) estimated the analog and digital distortions of satellite signals using high-gain antennas and assessed their impacts using a receiver performance model. Liu et al. (2019b) proposed a model for the effect of group delay variation characteristics on pseudorange biases. Vergara et al. (2020) presented a model for the DLL tracking performance and derived an expression of the distorted discriminators, from which the zero-crossing point bias (namely, the pseudorange bias) can be determined. Even with these theoretical models, the pseudorange biases have to be calculated from the $\mathrm{CCF}$ or discriminator function by means of iteration and/or numerical simulation. On the other hand, it is often assumed that a channel without pseudorange biases should be a linear phase system.

We present two sufficient conditions for pseudorange-bias-free linear channel characteristics and their implementation methods and comparatively analyze their effects, providing a theoretical foundation for eliminating pseudorange biases. The equation of ideal channel characteristics for the goal of "no pseudorange bias" is first derived by using the equivalent baseband model. We found that in addition to requiring the entire channel to be a linear phase system, the channel with cascaded even-symmetric phase, linear phase, and even-symmetric amplitude responses can also be free of pseudorange biases. Second, the Abel-Smith all-pass filter is introduced to digitally compensate the channel phase characteristics according to the two sufficient conditions (two criteria), whose performance is optimized and evaluated with the goal of minimum S-curve bias (SCB). Third, through the simulation of BPSK(10) signal, the remarkable effect of the designed phase equalizer in eliminating biases is verified, and the complexity and compensation results of the two criteria are compared. Finally, the live BDS3 PRN32 and PRN33 satellite B1C signals collected by a high-gain parabolic antenna are utilized to invert the real channel characteristics and then sent to the accordingly constructed phase equalizers before being processed by our software receiver. The measurement results demonstrate that the channel phase equalizers following the above two criteria can significantly reduce the pseudorange biases. In practice, either criterion can be selected for phase equalizer design according to different channel conditions and design requirements, which can effectively solve the pseudorange bias problem and is suitable for various signal modulations and high-accuracy applications.

\section{Theoretical model of the transfer function of pseudorange-bias-free linear channel}

Pseudorange biases are caused by satellite signal code chip waveform distortions. If the ideal signal is directly fed into the digital correlator of a well-designed receiver, there will be no systematic bias in pseudorange and carrier phase measurements in the absence of multipath and interference. In reality, however, the input of the digital correlator is a corrupted signal because the ideal signal will go through a physical channel formed by a cascade of nonideal components. The channel is mainly composed of the following three parts: (1) The satellite signal generation channel in the payload; (2) the wireless signal propagation channel from the phase center of the satellite transmitting antenna to the phase center of the receiver antenna; (3) the receiver channel from the antenna port to the digital correlator entrance. 
The nonidealities of these components may lead to two types of signal distortions: linear distortions and nonlinear distortions (Soellner et al. 2002). We will try to derive the requirement for the linearized system to obtain unbiased measurements. This is meaningful for the following reasons: (1) It will provide theoretical guidance for the design optimization of linear components and the entire channel, even for channels with nonlinear components which are usually linearized in theoretical analysis and practical implementation; (2) linear systems can be analyzed by using mathematical tools for signal analysis (such as impulse response and frequency response), whereas it is usually difficult to use analytical methods rather than simulation methods to evaluate the effects of nonlinear distortions caused by high power amplifier (HPA) with high-order nonlinearity in the payload; (3) linear filters can often be used to remove noise and distortions created by nonlinear processes.

The ideal, undistorted radio frequency (RF) GNSS signal can be modeled as $\sqrt{P} s(t) e^{j 2 \pi f_{c} t}$, where $P$ is the RF signal power, $f_{c}$ is the carrier frequency, $s(t)$ is the baseband complex envelope with normalized power in the low-pass equivalent model (Proakis and Salehi 2008), which is composed of $N$ signal components $b_{n}(t)$ (usually binary) $(n=1,2, \ldots, N)$, with their corresponding power coefficients $\alpha_{n}$ and phase assignments $\theta_{n}$ :

$s(t)=\sum_{n=1}^{N} \sqrt{\alpha_{n}} b_{n}(t) e^{j \theta_{n}}$

Usually, $s(t)$ is designed as a constant envelope signal in order to mitigate the nonlinear distortion induced by HPA in the payload signal generation chain. Ideally, any two different components, such as $b_{n}(t) e^{j \theta_{n}}$ and $b_{m}(t) e^{j \theta_{m}}$, should be orthogonal, which means that the cross-correlation between them should be zero. This goal can be approached as close as possible by carefully designing the spread spectrum code structure and the carrier phase relationship of different signal components. We mainly study the nonideal channel transfer function effects on the correlation function, assuming that different signal components are orthogonal.
Let us focus on the $n^{\text {th }}$ useful signal component tracked by the receiver DLL. Assuming that the receiver carrier tracking loop is tracking the exact carrier phase, we can obtain the lowpass (baseband) equivalent model of the linear channel and receiver correlator as shown in Fig. 1.

The linear channel can be equivalent to a low-pass filter described by its impulse response $h(t)$ and frequency response $H(\omega)$, which constitute a pair of Fourier transforms. If the filter phase response is linear, the filter will delay signals with different frequencies for the same length of time. In order to facilitate the analysis, $H(\omega)$ can be theoretically divided into the following cascade of two filters: The first stage is an ideal filter $H_{1}(\omega)$ with a constant amplitude 1 , a fixed delay $\tau_{d}$, and a fixed phase shift $\varphi_{d}$, while the second stage is a nonideal filter $H_{2}(\omega)$ with a phase-frequency response $\varphi_{2}(\omega)$ and an amplitude-frequency response $\left|H_{2}(\omega)\right|$, as shown in:

$H(\omega)=H_{1}(\omega) H_{2}(\omega)$

$H(\omega)=|H(\omega)| e^{j \varphi(\omega)}=|H(\omega)| e^{-j \int g(\omega) d \omega}$

$H_{1}(\omega)=e^{j\left(\omega \tau_{d}+\varphi_{d}\right)}$

$H_{2}(\omega)=\left|H_{2}(\omega)\right| e^{j \varphi_{2}(\omega)}=\left|H_{2}(\omega)\right| e^{-j \int g_{2}(\omega) d \omega}$

where $g(\omega)=-\frac{d \varphi(\omega)}{d \omega}$ is the group delay response of the entire channel and $g_{2}(\omega)=-\frac{d \varphi_{2}(\omega)}{d \omega}$ is the group delay response of the second-stage filter with its fixed constant part incorporated into the previous $\tau_{d}$ term. Thus, in our analysis model, $\tau_{d}$ represents the true code phase delay and $g_{2}(\omega)$ accounts for the nonideal group delay response part that may give rise to pseudorange bias.

The output signals of the two filters are

$s_{1}(t)=s\left(t-\tau_{d}\right) e^{j \varphi_{d}}=\sum_{n=1}^{N} \sqrt{\alpha_{n}} b_{n}\left(t-\tau_{d}\right) e^{j\left(\theta_{n}+\varphi_{d}\right)}$

$s_{2}(t)=s_{1}(t) * h_{2}(t)$

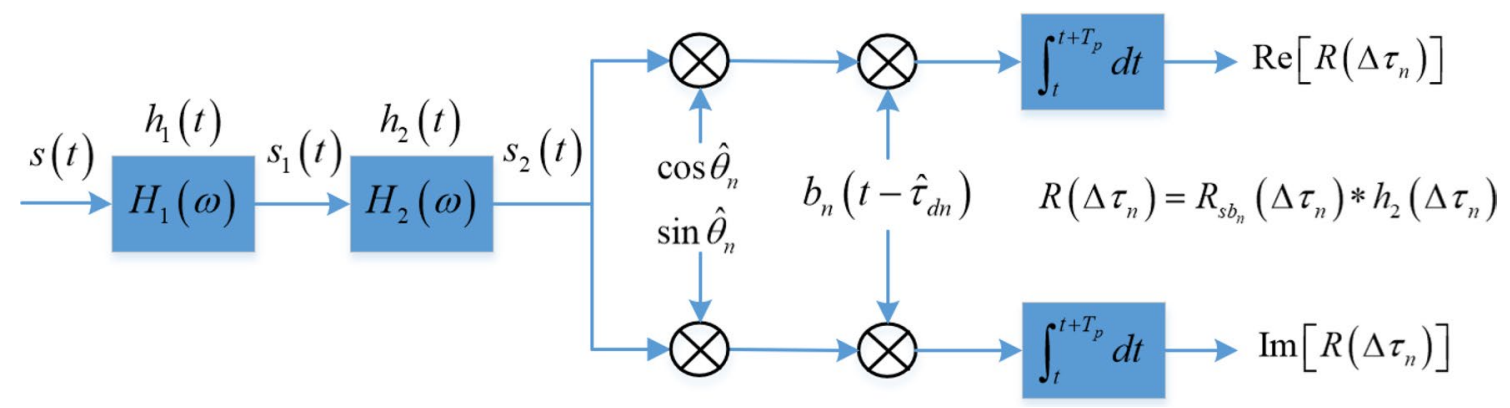

Fig. 1 Equivalent baseband model of satellite-receiver linear channel and receiver correlator. The carrier phase tracking error is ignored 
where the symbol * represents the convolution operation. The correlator output of the $n$th signal component is the cross-correlation of $s_{2}(t)$ and the local replica code signal $b_{n}\left(t-\hat{\tau}_{d n}\right)$ :

$$
\begin{aligned}
R\left(\Delta \tau_{n}\right)= & {\left[s_{1}(t) * h_{2}(t)\right] \otimes b_{n}\left(t-\hat{\tau}_{d n}\right)=R_{s_{1} b_{n}}\left(\Delta \tau_{n}\right) * h_{2}\left(\Delta \tau_{n}\right) } \\
= & \sqrt{\alpha_{n}}\left[R_{n}\left(\Delta \tau_{n}\right) * h_{2}\left(\Delta \tau_{n}\right)\right] \operatorname{sinc}\left(\pi \Delta f_{n} T_{p}\right) e^{j \Delta \theta_{n}} \\
& +\sum_{\substack{m=1 \\
m \neq n}}^{N} \sqrt{\alpha_{m}}\left[R_{m n}\left(\Delta \tau_{n}\right) * h_{2}\left(\Delta \tau_{n}\right)\right] \operatorname{sinc}\left(\pi \Delta f_{n} T_{p}\right) e^{j \Delta \theta_{n}}
\end{aligned}
$$

$R_{n}\left(\Delta \tau_{n}\right)=\frac{1}{T_{p}} \int_{t}^{t+T_{p}} b_{n}\left(t-\tau_{d}\right) b_{n}\left(t-\hat{\tau}_{d n}\right) \mathrm{d} t$

where $\hat{\tau}_{d n}$ is the delay of the local replica code signal, which is the estimate of $\tau_{d}$ by the code loop for the $n$th signal component, $\Delta \tau_{n}=\hat{\tau}_{d n}-\tau_{d}$ is the code phase estimation error, $\Delta \theta_{n}=\hat{\theta}_{n}-\theta_{n}-\varphi_{d}$ is the carrier phase estimation error, $\Delta f_{n}$ is the carrier Doppler frequency error of the carrier tracking loop for the $n$th signal component, and $T_{p}$ is the coherent

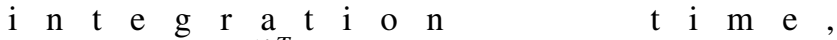
$R_{m n}\left(\Delta \tau_{n}\right)=\frac{1}{T_{p}} \int_{t}^{t+T_{p}} b_{m}\left(t-\tau_{d}\right) b_{n}\left(t-\hat{\tau}_{d n}\right) \mathrm{d} t$ is the CCF between the $m$ th component $b_{m}\left(t-\tau_{d}\right)$ of the incoming signal and the locally generated $n$th signal component $b_{n}\left(t-\hat{\tau}_{d n}\right)$, and $R_{n n}\left(\Delta \tau_{n}\right)$ is simply denoted as $R_{n}\left(\Delta \tau_{n}\right)$. The expanded form of $R_{s_{1} b_{n}}\left(\Delta \tau_{n}\right)$ in (8) can be derived in a similar way to Eqs. (49) and (50) in Van Dierendonck (1996).

Ignoring the CCF $R_{m n}\left(\Delta \tau_{n}\right)(m \neq n)$ between different signal components, we have

$R\left(\Delta \tau_{n}\right)=\sqrt{\alpha_{n}}\left[R_{n}\left(\Delta \tau_{n}\right) * h_{2}\left(\Delta \tau_{n}\right)\right] \operatorname{sinc}\left(\pi \Delta f_{n} T_{p}\right) e^{j \Delta \theta_{n}}$

Ignoring the carrier phase error $\Delta \theta_{n}$ and carrier frequency error $\Delta f_{n}$, the correlation output is further simplified as

$R\left(\Delta \tau_{n}\right)=\sqrt{\alpha_{n}}\left[R_{n}\left(\Delta \tau_{n}\right) * h_{2}\left(\Delta \tau_{n}\right)\right]$

Accordingly, its Fourier transform is

$G(\omega)=\sqrt{\alpha_{n}} G_{n}(\omega) H_{2}(\omega)$

where $G_{n}(\omega)$ is the power spectral density (PSD) of $b_{n}(t)$.

Even if the CCF is simplified to the form of (11), the calculation from the channel characteristic $H(\omega)$ or phase response $\varphi(\omega)$ to the lock point bias is still nonlinear, so the influence of the cascade channel on pseudorange bias cannot merely be taken for granted as a linear superposition of the influence of each stage.

Zero pseudorange bias means zero bias of the S-curve zero-crossing point, that is, the DLL locks to the point where the local code phase $\left(t-\hat{\tau}_{d n}\right)$ is aligned with the true incoming code phase $\left(t-\tau_{d}\right)$. From the DLL principle, it can be known that the necessary and sufficient condition for the pseudorange bias to be zero is that the CCF incorporated into the discriminator should be even-symmetric with respect to its code phase error.

For a DLL employing a coherent/quasi-coherent dot product power discriminator (Ward 2017), it requires that

$\operatorname{Re}\left[R\left(-\Delta \tau_{n}\right)\right]-\operatorname{Re}\left[R\left(\Delta \tau_{n}\right)\right]=0$

Since the autocorrelation function $R_{n}\left(\Delta \tau_{n}\right)$ and PSD $G_{n}(\omega)$ of the real signal $b_{n}(t)$ are real even functions, accordingly the Fourier transform of $\operatorname{Re}\left[R\left(\Delta \tau_{n}\right)\right]$ is

$\frac{1}{2}\left[G(\omega)+G^{*}(-\omega)\right]=\frac{1}{2} G_{n}(\omega)\left[H_{2}(\omega)+H_{2}^{*}(-\omega)\right]$

Thus the imaginary part of the channel transfer function should satisfy:

$\operatorname{Im}\left[H_{2}(-\omega)\right]=\operatorname{Im}\left[H_{2}(\omega)\right]$

This is the necessary and sufficient condition of the channel with zero pseudorange bias for coherent DLL with arbitrary correlator spacing for any real signal component, which requires the imaginary part of the channel transfer function to be even-symmetric. In the actual band-pass system, such even symmetry is centered on the nominal carrier frequency.

In practice, noncoherent discriminators are more widely used because of their robustness to data modulation and carrier tracking errors. For a noncoherent discriminator such as an early-minus-late power/envelope (EMLP/EMLE) discriminator, the requirement becomes

$\left|R\left(-\Delta \tau_{n}\right)\right|^{2}-\left|R\left(\Delta \tau_{n}\right)\right|^{2}=0$

$R^{*}\left(-\Delta \tau_{n}\right) R\left(-\Delta \tau_{n}\right)-R^{*}\left(\Delta \tau_{n}\right) R\left(\Delta \tau_{n}\right)=0$

$\left[R_{n}\left(\Delta \tau_{n}\right) * h_{2}^{*}\left(-\Delta \tau_{n}\right)\right]\left[R_{n}\left(\Delta \tau_{n}\right) * h_{2}\left(-\Delta \tau_{n}\right)\right]$
$\quad-\left[R_{n}\left(\Delta \tau_{n}\right) * h_{2}^{*}\left(\Delta \tau_{n}\right)\right]\left[R_{n}\left(\Delta \tau_{n}\right) * h_{2}\left(\Delta \tau_{n}\right)\right]=0$

$\left[G_{n}(\omega) H_{2}^{*}(\omega)\right] *\left[G_{n}(\omega) H_{2}(-\omega)\right]$

- $\left[G_{n}(\omega) H_{2}^{*}(-\omega)\right] *\left[G_{n}(\omega) H_{2}(\omega)\right]=0$

The left side of (19) is illustrated in Fig. 2 to facilitate analysis.

From Fig. 2, we can derive two sufficient conditions of the channel with zero pseudorange bias for noncoherent DLL, as shown in Table 1. Sufficient condition 1 means that the entire channel is a linear phase (constant group delay) system with an arbitrary amplitude-frequency response. Sufficient condition 2 implies that the entire channel has an even-symmetric amplitude-frequency response and a phase-frequency response composed of 


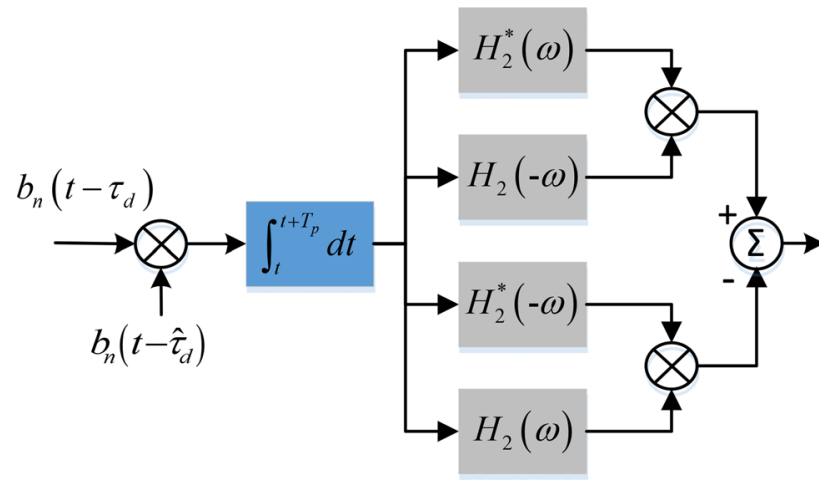

Fig. 2 Condition of pseudorange-bias-free channel for noncoherent DLL. The functions in gray boxes represent frequency domain transfer functions

linear and even-symmetric term. Sufficient conditions 1 and 2 are also two special cases of zero-pseudorange-bias channel in (15) for coherent/quasi-coherent DLL.

Therefore, these two sufficient conditions form the theoretical model of pseudorange-bias-free linear channel and serve as two criteria for designing the linear channel transfer function with minimum pseudorange bias. They are independent of signal structures and applications and do not limit receiver front-end bandwidth and correlator spacing.

\section{Simulation of pseudorange biases of cascade channel with different frequency responses}

We will take the BPSK(10) modulation, which has been widely adopted in GNSS, as an example to verify by simulation the two sufficient conditions for pseudorange-bias-free channel in Table 1. The influence of different amplitude- and phase-frequency responses of the linear channel on pseudorange biases, including its relationship with the influence of each stage of the cascade channel, will also be simulated.

\section{Two sufficient conditions of pseudorange-bias-free channel}

First, the simulation will check whether the pseudorange bias measured by the receiver will approach zero after an ideal, undistorted signal passes through a channel meeting condition 1 or condition 2 in Table 1 . In most cases, the group delay characteristics of practical filters can be expressed in polynomial form based on the Taylor series expansion (Zhu et al. 2008). Following the analysis of Liu et al. (2019b), we use a third-order polynomial for the group delay response and a fourth-order polynomial for the phase response in the simulation, as shown in (20) and (21), respectively.

$g(f)=\tau_{g 0}+2 \pi \tau_{g_{1}} f+2 \pi^{2} \tau_{g_{2}} f^{2}+\frac{4}{3} \pi^{3} \tau_{g_{3}} f^{3}$

$$
\begin{aligned}
\varphi(f)= & -2 \pi \tau_{g 0} f-\frac{1}{2}(2 \pi)^{2} \tau_{g 1} f^{2} \\
& -\frac{1}{6}(2 \pi)^{3} \tau_{g 2} f^{3}-\frac{1}{24}(2 \pi)^{4} \tau_{g 3} f^{4}
\end{aligned}
$$

where

$\tau_{g_{0}}=2.35 \mathrm{e}-8(\mathrm{~s}), \quad \tau_{g_{1}}=1.8 \mathrm{e}-17\left(\mathrm{~s}^{2} / \mathrm{rad}\right)$,

$\tau_{g_{2}}=1.75 e-24\left(\mathrm{~s}^{3} / \mathrm{rad}^{2}\right), \quad \tau_{g_{3}}=4.2 e-33\left(\mathrm{~s}^{4} / \mathrm{rad}^{3}\right)$

are the zeroth-, first-, second-, and third-order group delays, respectively. The values of these parameters are also from Liu et al. (2019b).

The simulated phase will take a combination of the following three terms:

$\varphi_{\operatorname{lin}}(f)=-2 \pi \tau_{g 0} f$

$\varphi_{\mathrm{even}}(f)=-\frac{1}{2}(2 \pi)^{2} \tau_{g 1} f^{2}-\frac{1}{24}(2 \pi)^{4} \tau_{g 3} f^{4}$

$\varphi_{\text {odd }}(f)=-\frac{1}{6}(2 \pi)^{3} \tau_{g 2} f^{3}$

As for the amplitude response $|H(f)|$, for simplicity, a combination of a constant value and a single-frequency
Table 1 Two sufficient conditions of pseudorange-biasfree channel for noncoherent DLL

\begin{tabular}{lll}
\hline Characteristics & Sufficient condition 1 & Sufficient condition 2 \\
\hline$H_{2}(\omega)$ & $H_{2}^{*}(\omega)=H_{2}(\omega)$, Real function & $H_{2}(-\omega)=H_{2}(\omega)$, Even function \\
$\left|H_{2}(\omega)\right|$ & Arbitrary function & Even function \\
$\varphi_{2}(\omega)$ & $k \pi$ & Even function \\
$g_{2}(\omega)$ & Zero & Odd function \\
$|H(\omega)|$ & Arbitrary function & Even function \\
$\varphi(\omega)$ & $\omega t_{d}+\theta$, Linear function & Linear plus even function \\
$g(\omega)$ & $t_{d}$, Constant value & Constant plus odd function \\
\hline
\end{tabular}


cosine/sine fluctuation are used to simulate its even-symmetric or asymmetric characteristics.

$A_{\text {even }}(f)=1+\alpha \cdot\left[\cos \left(2 \pi v_{p} f\right)-1\right]$

$A_{\text {asym }}(f)=1+\alpha \cdot\left[\sin \left(2 \pi v_{p} f\right)-1\right]$

where $\alpha=\frac{1}{2}\left(1-10^{-A / 20}\right), A(\mathrm{~dB})$ is the peak-to-peak value, and $v_{p}=5 \mathrm{e}-7(\mathrm{~s})$ is the period of the amplitude ripple.

The following three cases will be simulated according to the phase characteristics $\varphi(f)$ :

1. Linear phase: $\varphi_{\text {lin }}(f)$

2. Linear plus even-symmetric phase: $\varphi_{\mathrm{le}}(f)=\varphi_{\mathrm{lin}}(f)+\varphi_{\mathrm{even}}(f)$

3. Linear plus odd-symmetric phase: $\varphi_{10}(f)=\varphi_{\operatorname{lin}}(f)+\varphi_{\text {odd }}(f)$

In each simulation case, the amplitude responses are $A_{\text {even }}(f)$ and $A_{\text {asym }}(f)$ with $A=1 \mathrm{~dB}, 2 \mathrm{~dB}, 4 \mathrm{~dB}$, totally six examples.

Many studies have shown that the receiver correlator spacing and front-end bandwidth have significant impacts on pseudorange biases. Generally, the wider the channel bandwidth, the more obvious the impact of the nonideal transfer function on biases. The simulated ideal signal passes through a filter with a double-side bandwidth of $40 \mathrm{MHz}$ and a transfer function of the above cases and then are correlated with a local replica code signal, from which the S-curve zero-crossing biases under different correlator spacing can be obtained. The simulated correlator spacing is in the range of 0.1 to 1 chip, with a step of 0.05 chip. Taking the lock point bias under the correlator spacing of 1 chip as the benchmark, by making differences between the biases under other correlator spacings and the benchmark, the pseudorange bias differences with respect to correlator spacing can be obtained as shown in Figs. 3, 4 and 5, and their variation ranges are the SCBs. The phase response value of the bottom plot of each figure is doubled relative to the top plot.

Figure 3 shows the pseudorange biases under a phase response of $\varphi_{\text {lin }}(f)$ (top) or $2 \varphi_{\text {lin }}(f)$ (bottom) with different amplitude responses. As long as the phase is linear, regardless of its slope with respect to frequency and the range and characteristics of amplitude variation with frequency, the pseudorange biases are around zero in the entire correlator spacing range. In other words, the SCBs are close to zero. What remains to be explained is that the biases shown in the figure vary within -0.5 to $0.1 \mathrm{~mm}$, which is mainly caused by the inevitable numerical error in the simulation.

Figure 4 shows the pseudorange biases under a phase response of $\varphi_{\mathrm{le}}(f)$ (top) or $2 \varphi_{\mathrm{le}}(f)$ (bottom) with different amplitude responses. When the phase-frequency response is a linear phase plus an even-symmetric phase, the SCBs are almost zero with even-symmetric amplitude responses
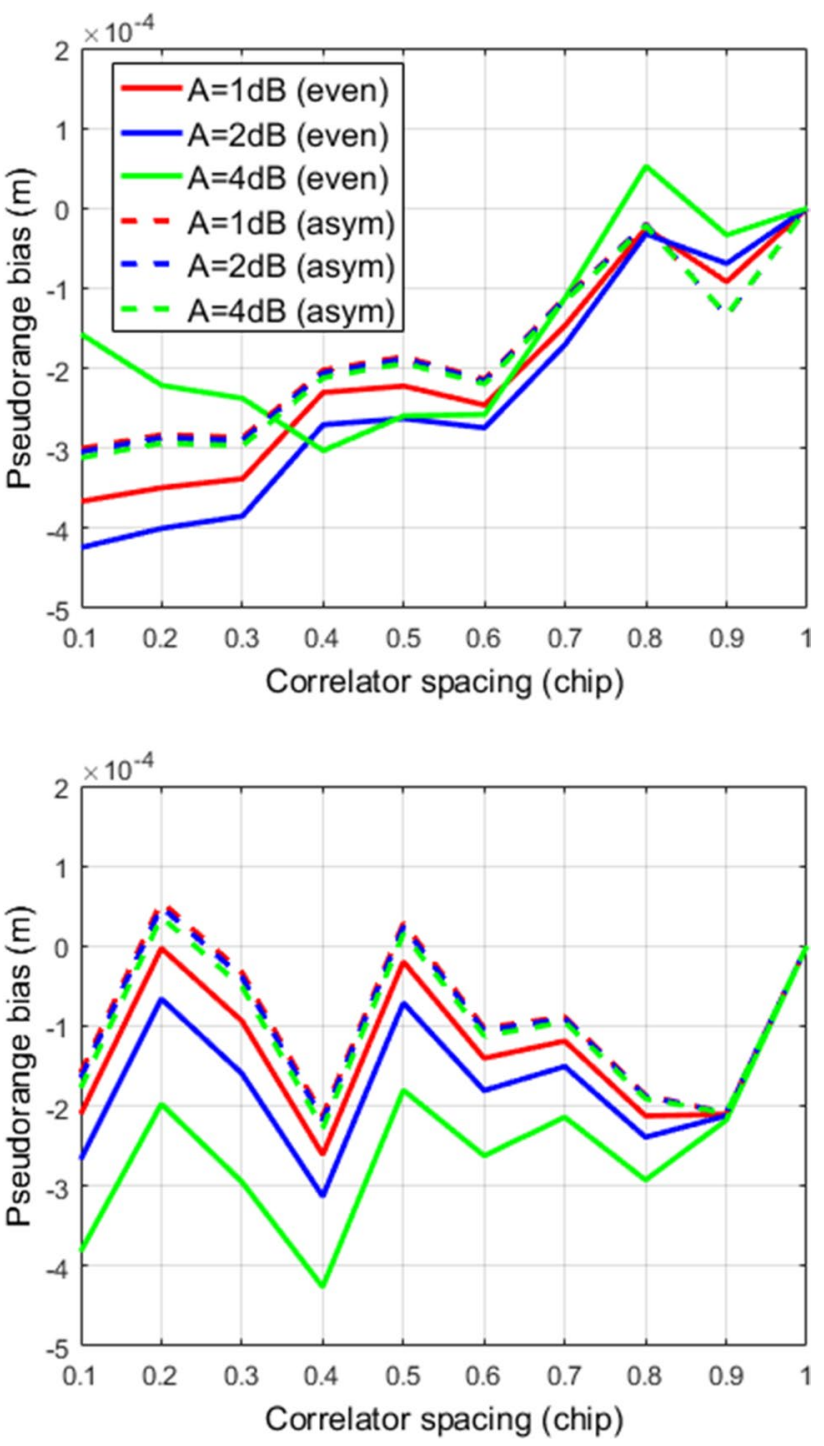

Fig. 3 Pseudorange biases for simulation case 1 with a linear phase of $\varphi_{\text {lin }}(f)$ (top) and $2 \varphi_{\text {lin }}(f)$ (bottom)

(as shown by the solid lines), while the SCBs are no longer close to zero with asymmetric amplitude responses (as shown by the dashed lines) because condition 2 is no longer satisfied. Nevertheless, the SCBs are still very small compared with the results of subsequent case 3 and increase as the amplitude response fluctuation range increases (from up to down in the two plots). Under the same asymmetric amplitude-frequency characteristics, the SCBs also increase correspondingly with the doubling of the symmetric phase response range (from the top to the bottom plot).

Figure 5 shows the pseudorange biases under a phase response of $\varphi_{\mathrm{lo}}(f)$ (top) and $2 \varphi_{\mathrm{lo}}(f)$ (bottom) with different amplitude responses. It can be seen that when the phase-frequency characteristic becomes a linear phase plus an odd-symmetric phase, the SCBs increase 

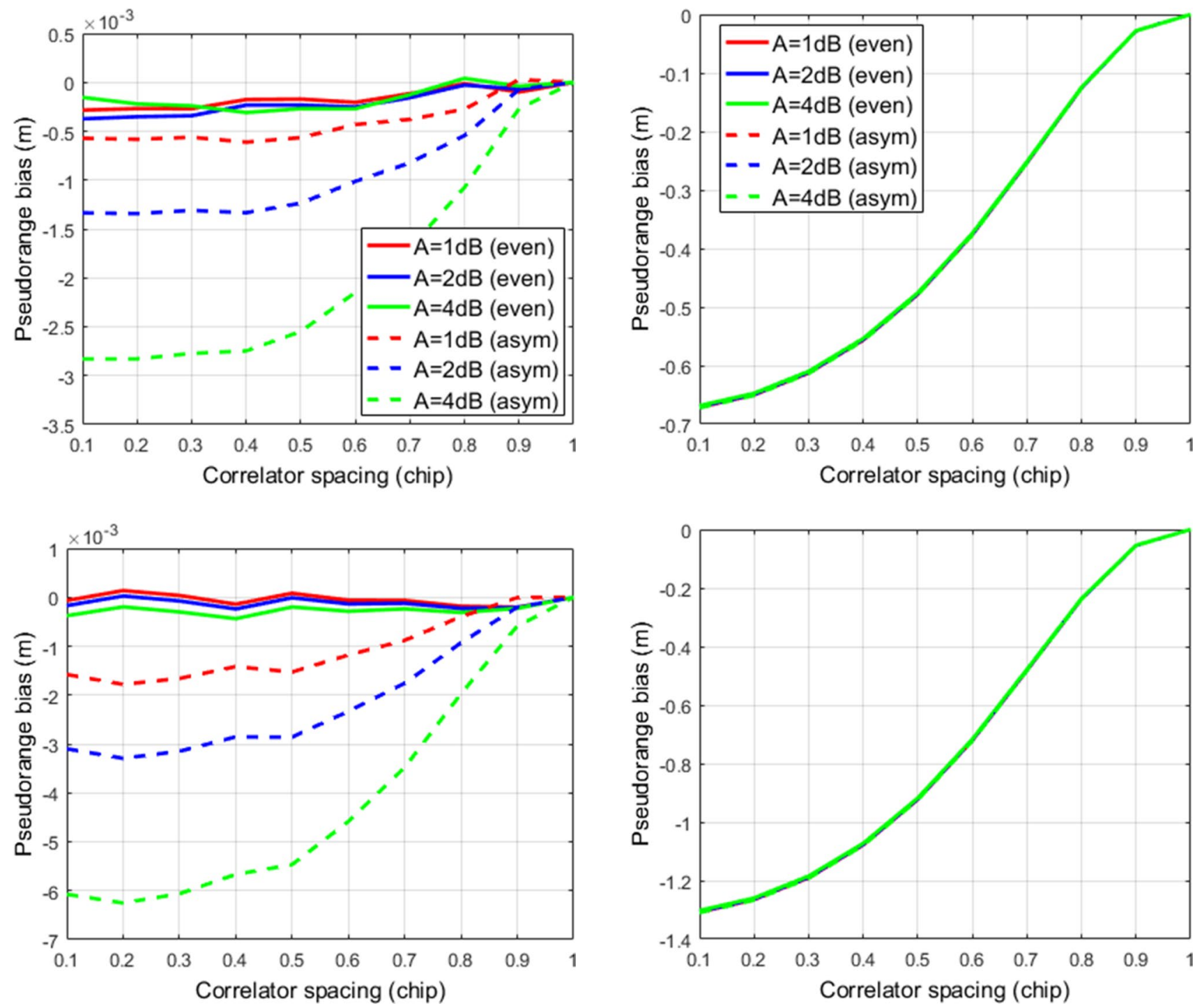

Fig. 4 Pseudorange biases for simulation case 2 with a linear plus even-symmetric phase of $\varphi_{\mathrm{le}}(f)$ (top) and $2 \varphi_{\mathrm{le}}(f)$ (bottom)

significantly and are obviously affected by the phase variation, whereas the influence of the amplitude characteristics is almost negligible compared with that of the phase characteristics.

In addition to verifying the correctness of our theoretical analysis, the simulation results in Figs. 3-5 also reveal that the influence of nonideal phase-frequency characteristics on pseudorange biases is much greater than that of nonideal amplitude-frequency characteristics. Although the significant influence of phase characteristics on pseudorange biases has been studied before, it is biased to assume that nonideal amplitude-frequency characteristics have no influence at all. Our simulation results for other types of phase-frequency and amplitude-frequency characteristics are also consistent with the above analysis.

Fig. 5 Pseudorange biases for simulation case 3 with a linear plus odd-symmetric phase of $\varphi_{\mathrm{lo}}(f)$ (top) and $2 \varphi_{\mathrm{lo}}(f)$ (bottom)

\section{Relationship between bias of cascade channel and sum of biases caused by each stage}

Here we use a simulation example to clarify the following point: The mechanism of pseudorange bias is the comprehensive effect of the nonideal characteristics of the satellite-receiver cascade channel, and the final bias is not merely the sum of the bias caused by the satellite alone and the bias caused by the receiver alone.

Leaving aside the phase nonlinearity of the wireless propagation channel for simplicity, its transfer function can be merged into $H_{1}(\omega)$ in Fig. 1 in our analysis model. Denote the transfer function of the satellite stage as $H_{\mathrm{s}}(\omega)=\left|H_{\mathrm{s}}(\omega)\right| e^{j \varphi_{s}(\omega)}$, and the transfer function of the 
receiver stage as $H_{\mathrm{r}}(\omega)=\left|H_{\mathrm{r}}(\omega)\right| e^{j \varphi_{r}(\omega)}$; then, the transfer function of the cascade channel from the satellite to the receiver is $H_{2}(\omega)=H_{\mathrm{s}}(\omega) H_{\mathrm{r}}(\omega)$.

According to the bias decomposition method in many traditional algorithms, the relationship between the pseudorange bias $\varepsilon(\delta)$ owing to the entire channel transfer function $H(\omega)$ and the bias $\varepsilon_{\mathrm{s}}(\delta)$ owing to the satellite stage transfer function $H_{\mathrm{s}}(\omega)$ and the bias $\varepsilon_{\mathrm{r}}(\delta)$ owing to the receiver stage transfer function $H_{\mathrm{r}}(\omega)$ should be like this: $\varepsilon(\delta)=\varepsilon_{\mathrm{s}}(\delta)+\varepsilon_{\mathrm{r}}(\delta)$, where $\delta$ is the correlator spacing. If we assume that the receiver stage transfer function satisfies the aforementioned sufficient condition 2, that is, $H_{\mathrm{r}}(-\omega)=H_{\mathrm{r}}(\omega)$, then $\varepsilon_{\mathrm{r}}(\delta)=0$ and $\varepsilon(\delta)=\varepsilon_{\mathrm{s}}(\delta)$. A simple simulation example in the following proves that this decomposition is incorrect.

In the simulation, the receiver bandwidth is $40 \mathrm{MHz}$, the receiver amplitude-frequency characteristic is constant, and the receiver phase-frequency response is parabolic. First, let us suppose that the satellite stage is an ideal linear phase system with a $40 \mathrm{MHz}$ bandwidth: $\quad \varphi_{s}(f)=k_{l} f, k_{l}=1.5 e-7(\mathrm{~s} \cdot \mathrm{rad}) ; \quad 1 \mathrm{et}$ $\varphi_{r}(f)=0, k f^{2}, 2 k f^{2}, 3 k f^{2}, 4 k f^{2}, \quad k=1.7 e-15\left(\mathrm{~s}^{2} \cdot \mathrm{rad}\right)$, respectively. After the simulated ideal BPSK(10) signal passes through the satellite-receiver cascade channel, its CCF with the ideal local replica code is computed, and the pseudorange biases incurred by signal distortions are extracted, as shown in Fig. 6 (top). Ignoring the numerical error of the simulation (less than $0.5 \mathrm{~mm}$ ), we have $\varepsilon(\delta)=\varepsilon_{\mathrm{s}}(\delta)=\varepsilon_{\mathrm{r}}(\delta)=0$.

Then the satellite channel is set as a fifth-order elliptic filter with a bandwidth of $40 \mathrm{MHz}$ and a passband fluctuation of $2 \mathrm{~dB}$, whereas the receiver stage still holds its evensymmetric phase as $\varphi_{r}(f)=0, k f^{2}, 2 k f^{2}, 3 k f^{2}, 4 k f^{2}$. After the ideal BPSK(10) signal passes through the cascade channel, the extracted pseudorange biases incurred by signal distortions are shown in Fig. 6 (bottom), where the solid red line can be seen as $\varepsilon_{\mathrm{s}}(\delta)$.

We can see that $\varepsilon(\delta) \neq \varepsilon_{\mathrm{s}}(\delta)$, although $\varepsilon_{\mathrm{r}}(\delta)=0$ is still valid for all the four cases of green, blue, orange, and purple lines. Whether the final pseudorange bias $\varepsilon(\delta)$ is increased or decreased from $\varepsilon_{\mathrm{s}}(\delta)$ depends on whether the receiver stage has aggravated or compensated somehow (such as $\varphi_{r}(f)=k f^{2}, 2 k f^{2}, 3 k f^{2}$ ) or overcompensated (such as $\varphi_{r}(f)=4 k f^{2}$ represented by the solid purple line) for the nonideal phase characteristics of the satellite-stage channel.

This simulation shows that $\varepsilon(\delta)=\varepsilon_{\mathrm{s}}(\delta)+\varepsilon_{\mathrm{r}}(\delta)$ is not true. The traditional processing method to decompose the pseudorange bias into the satellite-specific bias and the receiverspecific bias needs to be corrected. Sometimes the effects of cascading stages add up constructively, and sometimes they compensate each other. Therefore, designing a channel equalizer in the receiver to compensate for the nonideal
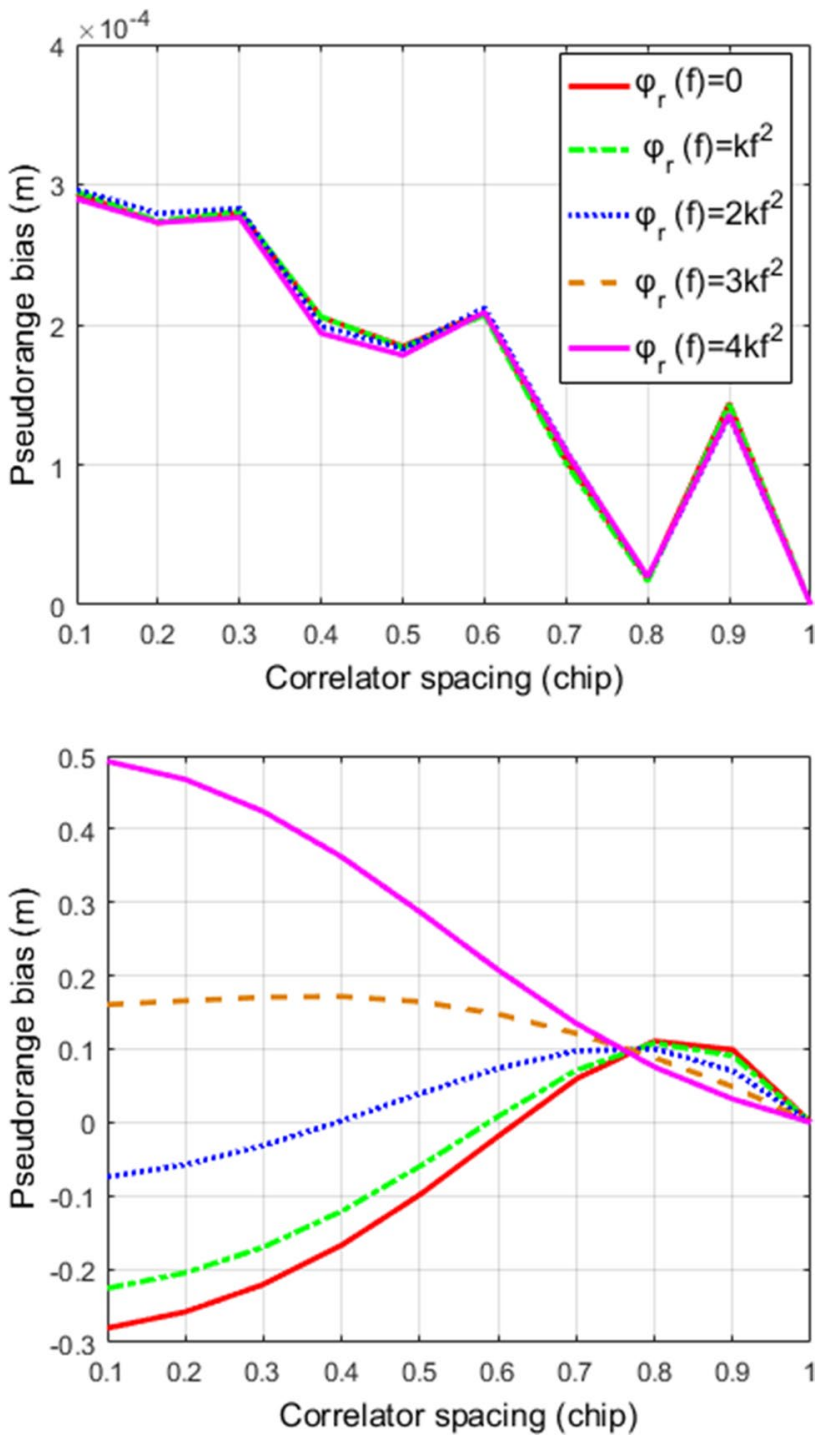

Fig. 6 Pseudorange biases for a cascade channel of a first-stage linear phase channel (top) and nonideal channel (bottom) plus a secondstage parabolic phase channel

transfer function of the satellite stage can reduce the pseudorange biases.

\section{Channel phase equalization method for eliminating pseudorange bias}

From the above analysis and actual channel characteristics, we know that the nonidealities of the channel transfer function bring about pseudorange biases, on which the influence of amplitude characteristics is much smaller than that of phase characteristics and can be ignored in most cases. In order to eliminate pseudorange bias, according to the two criteria in Table 1, the phase-frequency characteristics of the channel can be compensated to a linear phase 
or the sum of a linear phase and an even-symmetric phase through a channel phase equalizer. This section mainly discusses how to construct a phase equalizer based on these two criteria and compares the compensation effects under the two criteria through simulation.

It is assumed in the simulation that the amplitude-frequency characteristics of the channel are constant, and the group delay response and phase response are the same as in (20)-(22), which are plotted in Fig. 7. Through the above analysis or simulation, it can be known that if the ideal BPSK(10) signal passes through this channel, the resulting pseudorange biases with respect to correlator spacing will follow the curve shown in Fig. 5 (top), with an SCB of about $0.67 \mathrm{~m}$.

Since the amplitude-frequency response of an all-pass filter is constant in the entire frequency domain, and the phase-frequency response is nonlinear, the all-pass filter is typically used to change the relative phase relationship of different frequency components. That is, the all-pass filter is used as a phase equalizer (group delay equalizer) to achieve the expected target phase characteristics without changing the channel amplitude characteristics. Abel and Smith (2006) proposed an all-pass filter construction method with low computational complexity and stable performance. It divides the expected group delay frequency response of the all-pass filter into $N$ subsections with equal area and then uses one firstorder all-pass filter (with an area of $2 \pi$ ) to fit each subsection. By cascading $N$ first-order filters, an $N$-order all-pass filter can be obtained to approximate the desired phase response of the equalizer in the entire frequency band (Abel and Smith 2006; Abel et al. 2010). The principle and specific method
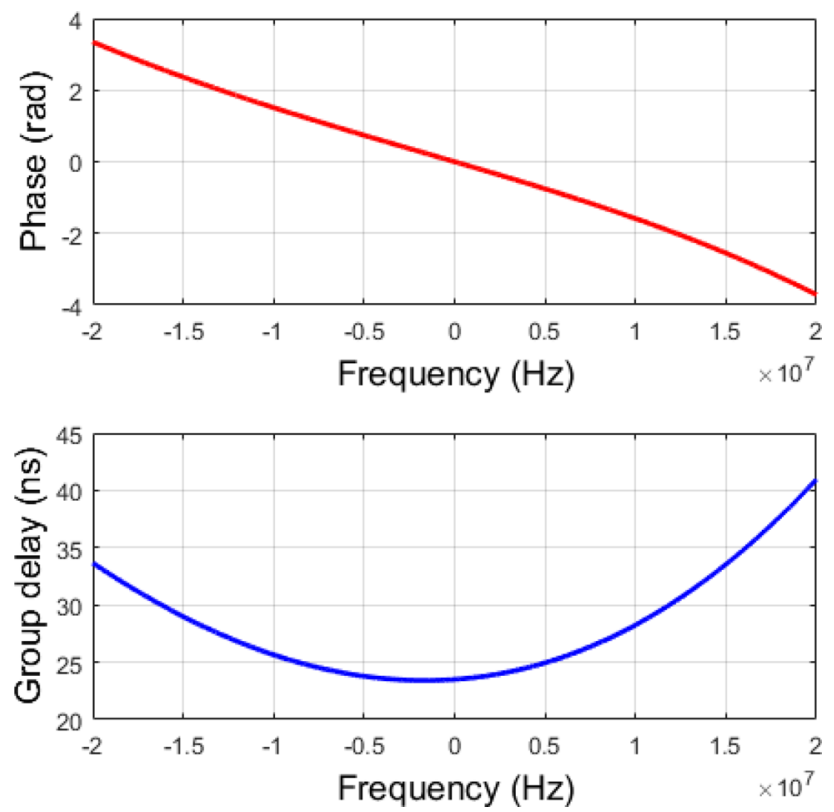

Fig. 7 Channel phase and group delay responses used in simulation and effects for compensating the distortions of GNSS signals caused by ionospheric dispersion have been studied in Guo et al. (2014). The method will be applied here, but according to Table 1, there are the following two target phase characteristic criteria.

\section{(1) Linear phase}

Denoting the group delay responses of the imperfect channel to be compensated, the desired target phase equalizer, and the target channel after compensation as $g(f), \tau_{e}(f)$, and $\tau_{c}(f)$, respectively, if the entire channel after adding the equalizer is expected to be an ideal linear phase system, then we have

$\tau_{c}(f)=g(f)+\tau_{e}(f)=C$

$\tau_{e}(f)=C-g(f)$

where the constant $C$ is introduced to ensure the engineering realizability of the equalization filter.

For the phase equalizer constructed by using the Abel-Smith all-pass filter, the area under the curve of the target group delay versus the normalized angular frequency is $2 \pi N$, namely

$\int_{0}^{0.5 f_{s}}[C-g(f)] \cdot d f=N$

where $f_{s}$ is the sampling frequency. So, the value of the constant $C$ can be obtained, which is related to the filter order $N$.

The Abel-Smith method needs to find the pole frequency and pole radius of the first-order all-pass filter corresponding to each subsection. Suppose the frequency interval of the $n$th subsection in the $N$ subsections is $\left[f_{n-1}, f_{n}\right]$, then

$\int_{f_{n-1}}^{f_{n}}[C-g(f)] d f=1$

Regarding $f_{n}$ as an independent variable, the value of each $f_{n}$ can be obtained by recursion starting from zero frequency.

The pole frequency of the first-order all-pass filter of each subsection is the midpoint of the frequency band:

$\Omega_{n}=\pi\left(f_{n-1}+f_{n}\right)$

Let $\beta$ be the ratio of the band edge group delay to the peak group delay of each first-order filter, whose value affects the smoothness of the final fitting, then the polar radius of each first-order filter is Abel and Smith 2006:

$r_{n}=\eta_{n}-\sqrt{\eta_{n}^{2}-1}$

where 

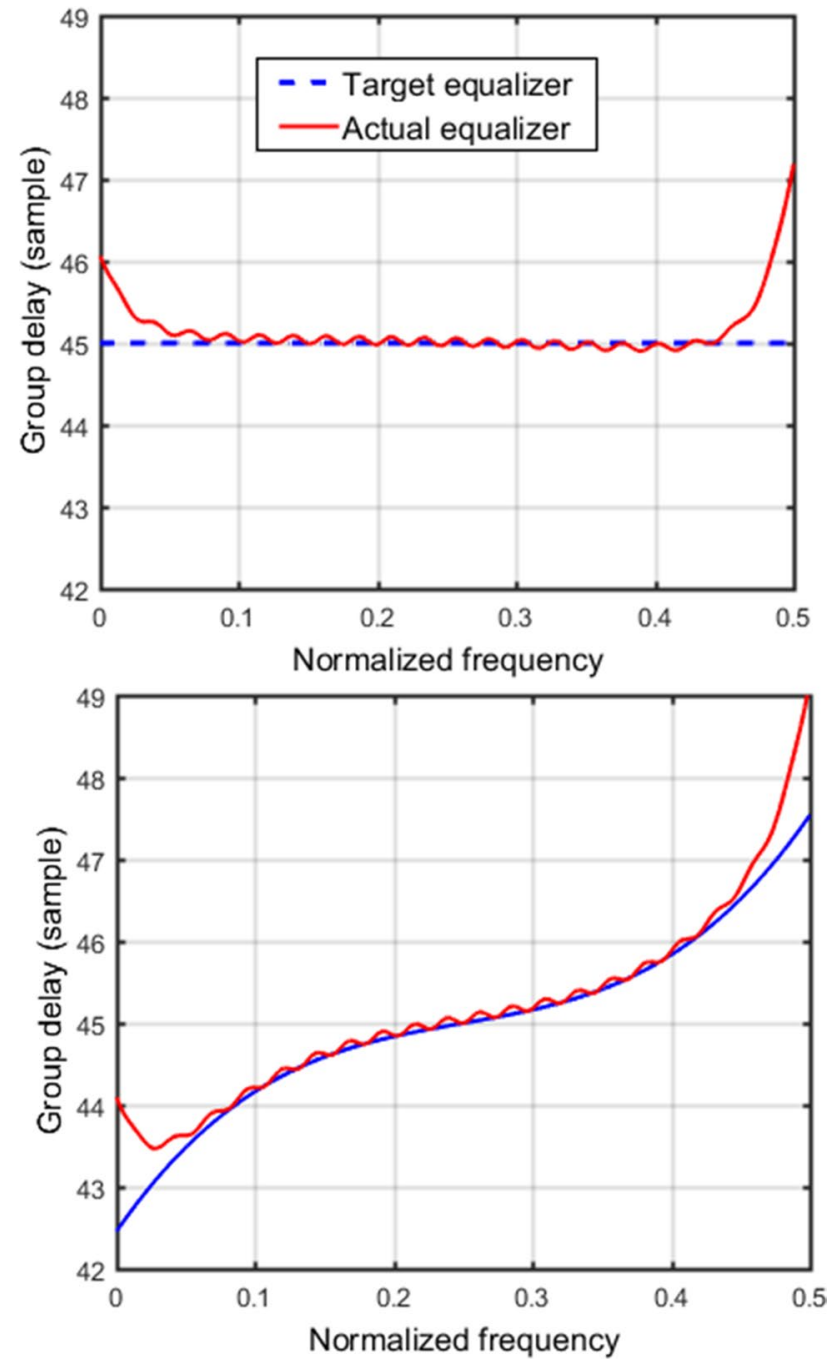

Fig. 8 Group delay compensation results with $N=20, \beta=0.85$ under criterion 1 with a linear phase (top) and criterion 2 with a linear plus even-symmetric phase (bottom)

$\eta_{n}=\frac{1-\beta \cos \Delta_{n}}{1-\beta}, \Delta_{n}=\pi\left(f_{n}-f_{n-1}\right)$

The pole of each subsection is $z_{n}=r_{n} e^{j 2 \pi \Omega_{n}}$.

Thus, the group delay of each first-order all-pass filter can be formed after calculating its pole frequency and pole radius, and the total group delay characteristic $\tau_{e a}(\omega)$ of the phase equalizer can be obtained by adding $\mathrm{N}$ group delays,

$\tau_{n}(\omega)=\frac{1-r_{n}^{2}}{1+r_{n}^{2}-2 r_{n} \cos \left(w-\Omega_{n}\right)}$

$\tau_{e a}(\omega)=\sum_{n=1}^{N} \tau_{n}(\omega)$ where the coefficients of each first-order all-pass filter are plurals. Each first-order all-pass filter can be cascaded by a counterpart filter with a complex conjugate pair of poles/ zeros to constitute a second-order all-pass filter, and then $N$ such second-order all-pass filters can be cascaded together to form an all-pass phase equalizer with real coefficients.

\section{(2) Linear phase plus even-symmetric phase}

Under this criterion, the target group delay frequency response of the entire channel is the sum of a constant and an odd function. If we express the group delay of the channel to be compensated as:

$g(f)=g_{\text {even }}(f)+g_{\text {odd }}(f)$

$g_{\text {even }}(f)=\frac{1}{2} g(f)+\frac{1}{2} g(-f)$

$g_{\text {odd }}(f)=\frac{1}{2} g(f)-\frac{1}{2} g(-f)$

then the target group delay response of the equalizer should be

$\tau_{e}(f)=\tau_{c}(f)-g(f)=C+\tau_{\text {odd }}(f)-g_{\text {even }}(f)$

where the constant $C$ together with the arbitrary odd function $\tau_{\text {odd }}(f)$ bring a lot of flexibility to the equalizer design, not necessarily complexity. If we fit $g(f)$ to a polynomial form, it is only necessary to compensate its even power terms to either a constant or an arbitrary odd function. For example, if $g(f)$ can be fitted to the third-order polynomial shown in (20), only the second-order term of the group delay response (corresponding to the third-order term of the phase response) needs to be compensated. We just take the following simulation example to compare the effects and complexity of the equalizers under criteria 1 and 2, leaving many other target function options allowed by (40) open for future study:

$\tau_{e}(f)=C-2 \pi^{2} \tau_{g_{2}} f^{2}$

After determining the target group delay characteristic, the construction method of the Abel-Smith phase equalizer is exactly the same as criterion 1 . The only difference between the two criteria lies in the target frequency response of the phase equalizer $\tau_{e}(f)$.

Since the phase characteristics of the all-pass filter are related to the values of $N$ and $\beta$, the equalizer has different compensation effects under different combinations of $N$ and $\beta$. The effects will be assessed in terms of the minimum mean square error (MSE) $\tau_{\mathrm{MSE}}$ of the actual group delay response $\tau_{e a}(f)$ relative to the target (expected) response 
Fig. 9 Compensation results in terms of group delay MSE with different $N$ and $\beta$ under criterion 1 with a linear phase (top) and criterion 2 with a linear plus even-symmetric phase (bottom) $\tau_{e}(f)$ of the equalizer and the post-compensation signal SCB, respectively:

$\tau_{\mathrm{MSE}}=\frac{1}{2 \pi B} \int_{0}^{2 \pi B}\left|\tau_{e a}(\omega)-\tau_{e}(\omega)\right|^{2} \mathrm{~d} \omega$

$\mathrm{SCB}=\max \left(\varepsilon_{\mathrm{bias}}(\delta)\right)-\min \left(\varepsilon_{\mathrm{bias}}(\delta)\right) \quad \delta \varepsilon[0.1,1]$

where $\delta$ is the correlator spacing, $\varepsilon_{\text {bias }}(\delta)$ is the pseudorange bias, and $B$ is the effective bandwidth of the equalization filter.

When $N=20$ and $\beta=0.85$, the group delay compensation results are shown in Fig. 8, where Fig. 8 (top) is the linear phase compensation result according to criterion 1 , and Fig. 8 (bottom) is the linear plus even-symmetric phase (the constant plus odd-symmetric group delay) compensation
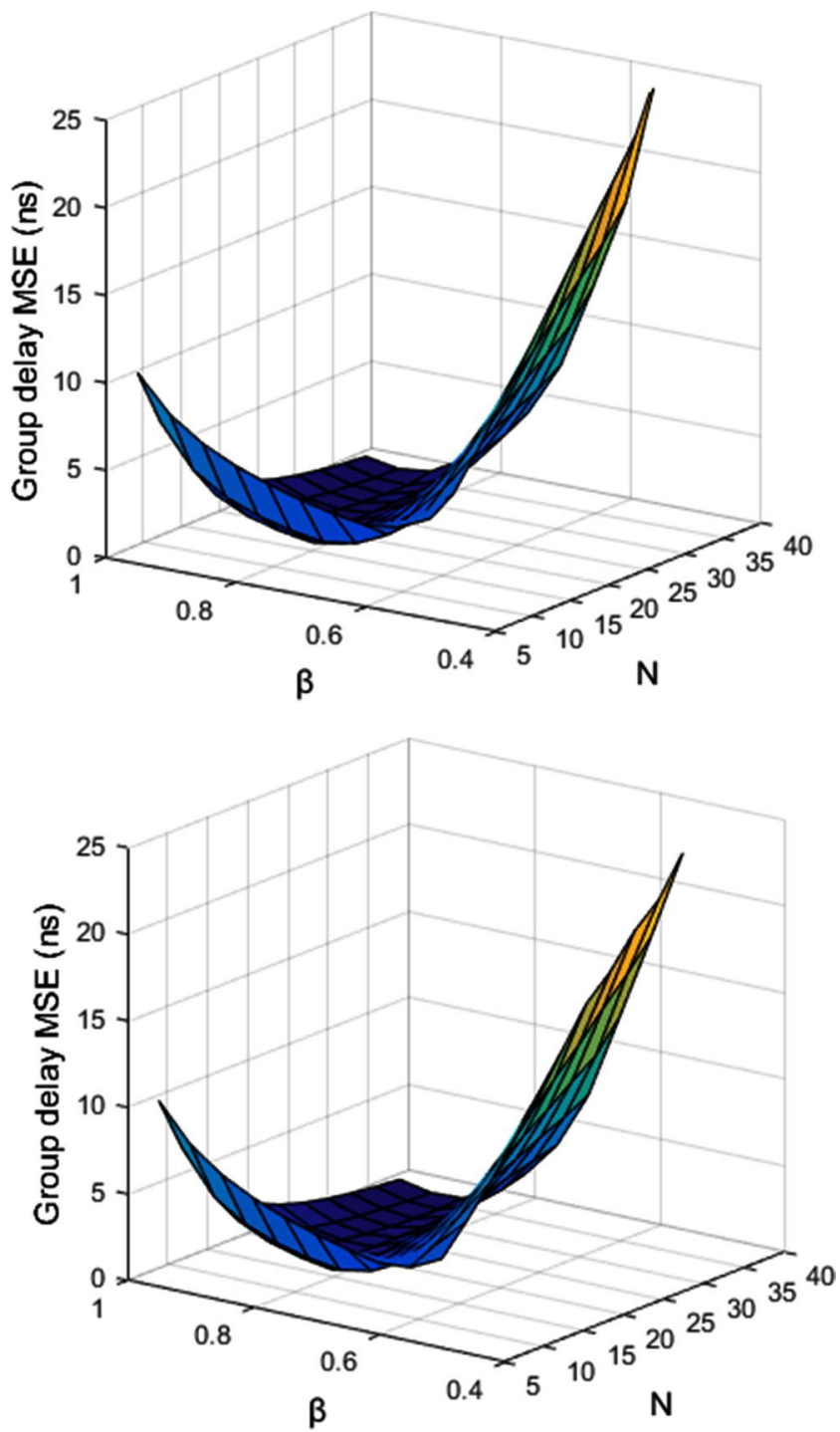

result according to criterion 2 . Figure 9 calculates the variation of $\tau_{\mathrm{MSE}}$ with different $N$ and $\beta$, where Fig. 9 (top) is based on criterion 1, and Fig. 9 (bottom) is based on criterion 2 .

The results in Fig. 9 can be used to optimize the parameters $N$ and $\beta$ according to $\tau_{\mathrm{MSE}}$. Of course, another important factor to be considered is that the order $N$ determines the complexity of the filter. It can be seen that when $N$ is small, $\tau_{\text {MSE }}$ first decreases with $\beta$ increasing and then gradually increases from about $\beta=0.8$; when $N$ is large, $\tau_{\text {MSE }}$ gradually decreases with $\beta$ increasing. The trend of $\tau_{\text {MSE }}$ changing with $N$ and $\beta$ under the two criteria is consistent, but the range of $\tau_{\mathrm{MSE}}$ under criterion 2 is slightly smaller than that under criterion 1 .

In order to further compare the compensation effects under the two criteria, taking parameter $\beta$ as the reference 

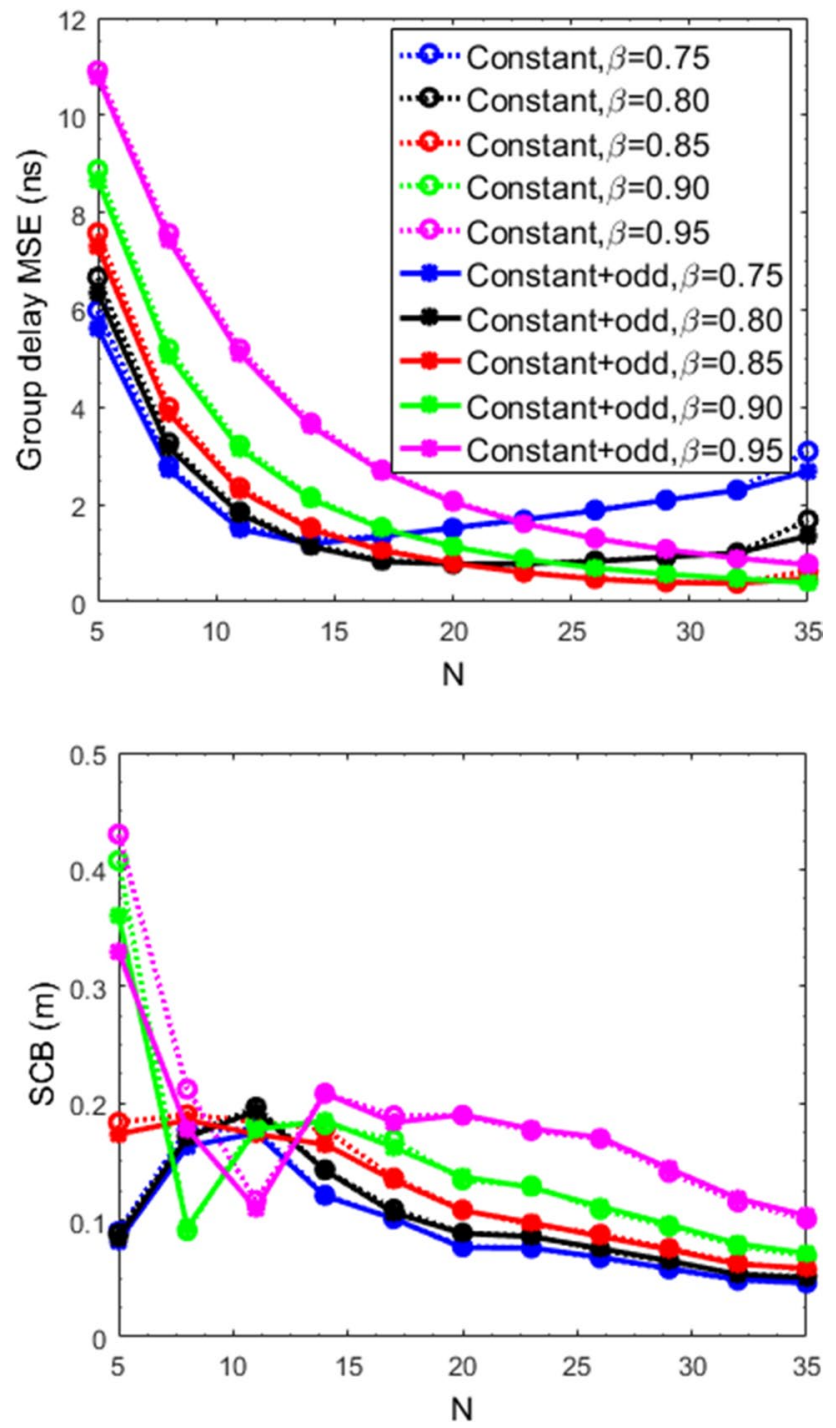

Fig. $10 \tau_{M S E}$ (top) and SCB versus $N$ with different $\beta$ (bottom). Results under criterion 1 are represented by dotted lines and $\mathrm{O}$ symbols, while those under criterion 2 are by solid lines and * symbols

variable and the filter cascade order $N$ as the independent variable, Fig. 10 simulates the equalizer group delay MSEs (top) and the SCBs of the compensated signal (bottom), where the dotted line and $\bigcirc$ symbol represent criterion 1 , and the solid line and $*$ symbol represent criterion 2 .

As can be seen, for the two compensation criteria, the group delay MSEs versus $N=5-32$ under each $\beta$ value have nearly identical values and trends. In some cases, the MSE under criterion 1 is slightly larger than under criterion 2 , but the SCB values under the two criteria almost show no difference. When the equalization filter order is low, the SCB performance of the signal obtained under the two compensation criteria is poor, but the effect of compensation for linear plus even-symmetric phase according to criterion 2 is slightly better than that for linear phase according to criterion 1 . With the order of the equalization filter increasing, the SCBs under the two criteria tend to be consistent. Under a $40 \mathrm{MHz}$ bandwidth, the SCB can be reduced from approximately $0.67 \mathrm{~m}$ for the uncompensated case to less than $0.1 \mathrm{~m}$ after employing the equalizer with $\beta=0.75$ and $N \geq 18$. It is worth noting that the SCB values and the level of improvement provided by the equalizer are also highly relevant to the channel bandwidth.

In principle, when using the Abel-Smith algorithm to design an equalizer for channel phase compensation, we can select the appropriate target equalizer phase characteristics under one of two criteria for phase compensation, according to different design requirements (such as order $N$ and compensation effect) and existing channel characteristics.

\section{BDS3 PRN32 and PRN33 satellite channel inversion and phase equalization}

This section performs channel estimation and phase equalization on the real satellite signals to verify the actual effect of the compensation method under the above two criteria. The test data come from the Haoping Observation Station of the National Time Service Center of the Chinese Academy of Sciences. The B1C signals transmitted by the BDS3 PRN32 and PRN33 satellites before their predistortion parameter uploading were received by a 40-m Cassegrain antenna and acquired by an intermediate frequency (IF) signal collector. Using the software receiver developed in our laboratory, the transfer functions of the two entire channels from the two satellite signal generation chains to the receiver RF front-end are estimated based on the correlationdomain method introduced in Soellner et al. (2008), which is also called the cross-power spectrum (CPS) inverse method and analyzed in Yan et al. (2018). Then by employing the Abel-Smith phase equalizers according to the two criteria of pseudorange-bias-free channel transfer function, we compensate the channel phase characteristics as linear or linear plus even-symmetric. The group delay characteristics before and after compensation are compared, and the pseudorange bias variations with correlator spacing are analyzed. The results for the PRN32 and PRN33 satellites are shown in the left and right plots of Figs. 11-14, respectively.

The high gain of the large-aperture antenna helps to improve the channel estimation precision. The IF of the collected digital signal is $62.5 \mathrm{MHz}$, and the sampling rate is $250 \mathrm{MHz}$. The software receiver acquires and tracks the collected satellite signals and correlates the incoming baseband signals (after carrier wipe-off) with the ideal local replica codes. In order to further eliminate the influence of noise on channel estimation, the CCF of each code period can be averaged as follows: 

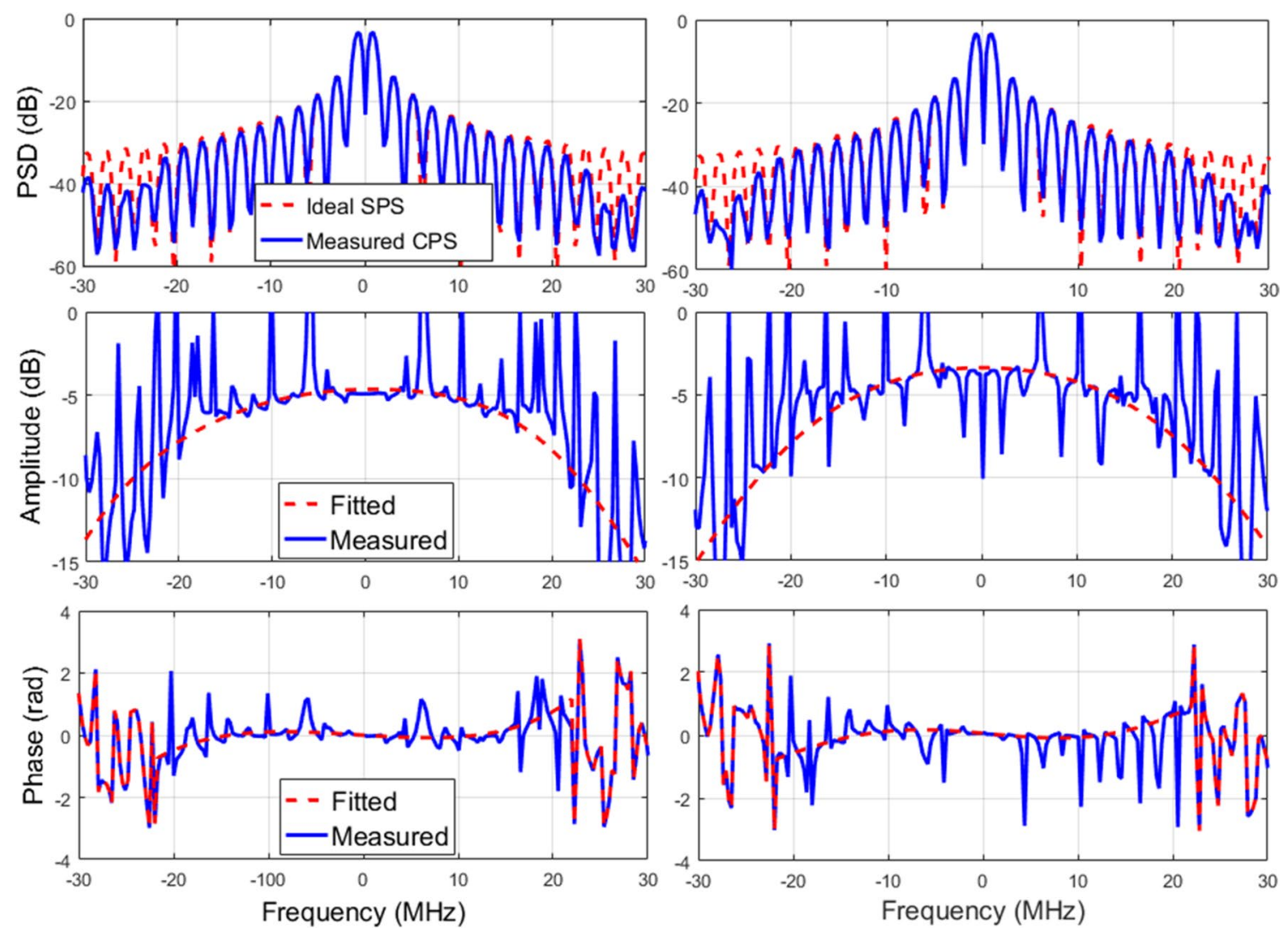

Fig. 11 Measured CPSs of BOC(1,1) components (top), measured and fitted amplitude (middle) and phase (bottom) responses of PRN32 (left) and PRN33 (right) satellite B1C channels

$\hat{R}_{x y}(\tau)=s_{x}(t) * \frac{1}{K} \sum_{i=1}^{K} s_{y}^{i}(t)=\frac{1}{K} \sum_{i=1}^{K} R_{x y}^{i}(\tau)$

The solid blue line in Fig. 11 (top panels) is the measured CPS $\hat{R}_{x y}(\tau)$ of the BOC $(1,1)$ component, where a clear PSD envelope can be seen. The channel amplitude and phase responses can be estimated by dividing the CPS of the measured signal by the auto-power spectrum (APS) of the ideal signal and performing appropriate denoising process, as shown in Fig. 11 middle and bottom panels respectively.

It can be seen from the amplitude responses that the channel $3 \mathrm{~dB}$ bandwidth of either satellite channel is about $37 \mathrm{MHz}$. The displayed channel phase responses are the results after removing the linear phase terms, and the dashed red lines are the fifth-order polynomial fitting results of the phase responses within the bandwidth:

$$
\begin{aligned}
\varphi(f)= & k_{5} \cdot f^{5}+k_{4} \cdot f^{4}+k_{3} \cdot f^{3} \\
& +k_{2} \cdot f^{2}+k_{1} \cdot f+k_{0}
\end{aligned}
$$

where for PRN32 satellite channel, $k_{5}=6.09 e-38\left(\mathrm{rad} \cdot \mathrm{s}^{5}\right)$, $k_{4}=4.47 e-31\left(\mathrm{rad} \cdot \mathrm{s}^{4}\right), k_{3}=1.01 e-22\left(\mathrm{rad} \cdot \mathrm{s}^{3}\right), k_{2}=1.68 e$ $-16\left(\mathrm{rad} \cdot \mathrm{s}^{2}\right), k_{1}=-1.86 e-8(\mathrm{rad} \cdot \mathrm{s}), k_{0}=1.83 e-3(\mathrm{rad}) ;$ forPRN33 satellite channel, $k_{5}=-9.34 e-38\left(\mathrm{rad} \cdot \mathrm{s}^{5}\right)$, $k_{4}=8.94 e-31\left(\mathrm{rad} \cdot \mathrm{s}^{4}\right), k_{3}=1.80 e-22\left(\mathrm{rad} \cdot \mathrm{s}^{3}\right), k_{2}=-4.33 e$ $-16\left(\mathrm{rad} \cdot \mathrm{s}^{2}\right), k_{1}=-2.71 e-8(\mathrm{rad} \cdot \mathrm{s}), k_{0}=6.15 e-2(\mathrm{rad})$. Accordingly, the group delay is: $g(f)=-\frac{1}{2 \pi} \cdot \frac{d \varphi(f)}{d f}$.

After measuring the channel phase response, we can use the Abel-Smith phase equalizer to compensate for the channel phase response as linear or linear plus even-symmetric. Setting the Abel-Smith filter parameters as $N=30$ and $\beta=0.87$, the pole-zero diagrams of the constructed linear phase equalizers are shown in Fig. 12. It can be seen that all the poles represented by the cross marks lie inside the unit circle, which ensures the stability of the designed filter. The channel group delay responses before and after compensation under the two criteria are shown in Fig. 13.

The pseudorange biases before and after compensation are measured by the software receiver to validate the effects of the phase equalizers. We take the receiver pseudorange measurement with a correlator spacing of 0.45 chip as the reference, obtain the differential pseudorange measurements with correlator spacings within 0.05 to 0.45 chip, and average them over a certain period of time (e.g. $1 \mathrm{~s})$ to suppress 
Fig. 12 Pole-zero diagrams of linear phase (constant group delay) equalizers (top) and linear plus even-symmetric phase (constant plus odd-symmetric group delay) equalizers (bottom) for PRN32 (left) and PRN33 (right) satellite B1C Channels
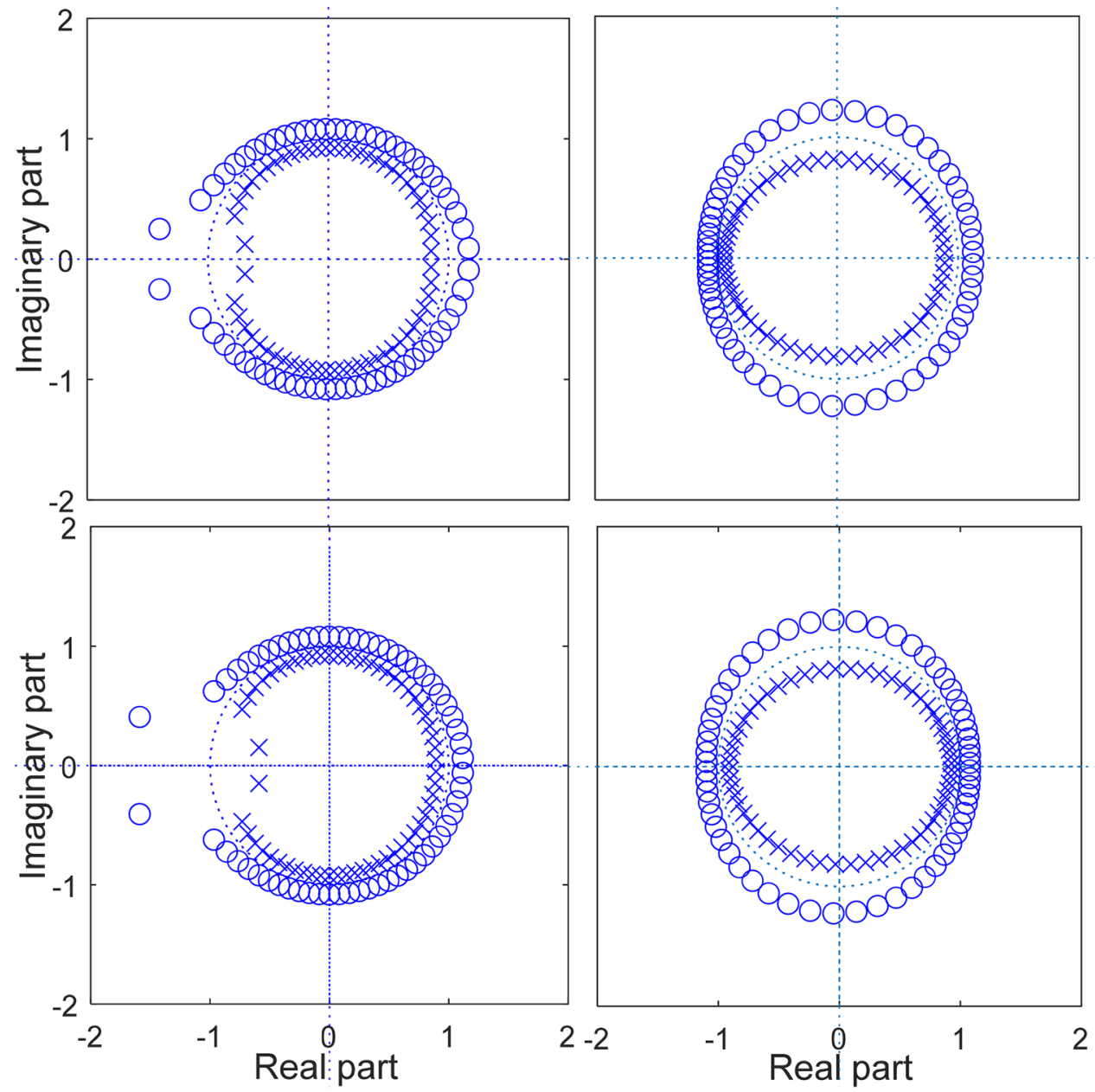

noises. The receiver-measured pseudorange biases with respect to correlator spacing are shown in Fig. 14.

Since the pseudorange biases are not only caused by the imperfect channel phase-frequency characteristics but also impacted by various other factors, they cannot be completely eliminated by channel phase compensation. However, the results show that the pseudorange biases can be significantly reduced by channel phase compensation under the two criteria. Before uploading the predistortion parameters, the nonideality of PRN32 satellite B1C channel is more severe than that of PRN33 satellite, and the resulting different pseudorange biases between different satellites under the same receiver processing cannot be eliminated by differential techniques. After linear phase compensation, the SCB is reduced from 0.65 to $0.226 \mathrm{~m}$ for PRN32 satellite and from 0.426 to $0.172 \mathrm{~m}$ for PRN33 satellite; while after linear plus even-symmetric phase compensation, the SCB is reduced to $0.194 \mathrm{~m}$ for PRN32 satellite and $0.173 \mathrm{~m}$ for PRN33 satellite. Therefore, the linear channel inversion and phase compensation method can effectively reduce the pseudorange biases.

\section{Conclusions}

With increasing diversity and bandwidth of satellite navigation signals, the pseudorange bias caused by nonideal characteristics of the satellite-receiver cascade channel has become a new core error source, which directly affects the service performance of satellite navigation systems and restricts their high-accuracy applications. We have provided a theoretical basis and design reference for solving the pseudorange bias problem for various signal modulations and applications from the perspective of signal channel improvement.

First, the ideal channel characteristics without pseudorange bias are derived through equivalent baseband analysis and verified by simulation, which indicates that: (1) When the entire cascade channel is a linear phase system, or when its phase-frequency characteristic is linear plus even-symmetric and its amplitude-frequency characteristic is evensymmetric, pseudorange biases will not be produced; (2) compared with amplitude characteristics, the effects of phase characteristics on pseudorange biases are much more 
Fig. 13 Channel group delay responses before and after compensation using linear phase (constant group delay) equalizers (top) and linear plus even-symmetric phase (constant plus odd-symmetric group delay) equalizers (bottom) for PRN32 (left) and PRN33 (right) satellite B1C channels
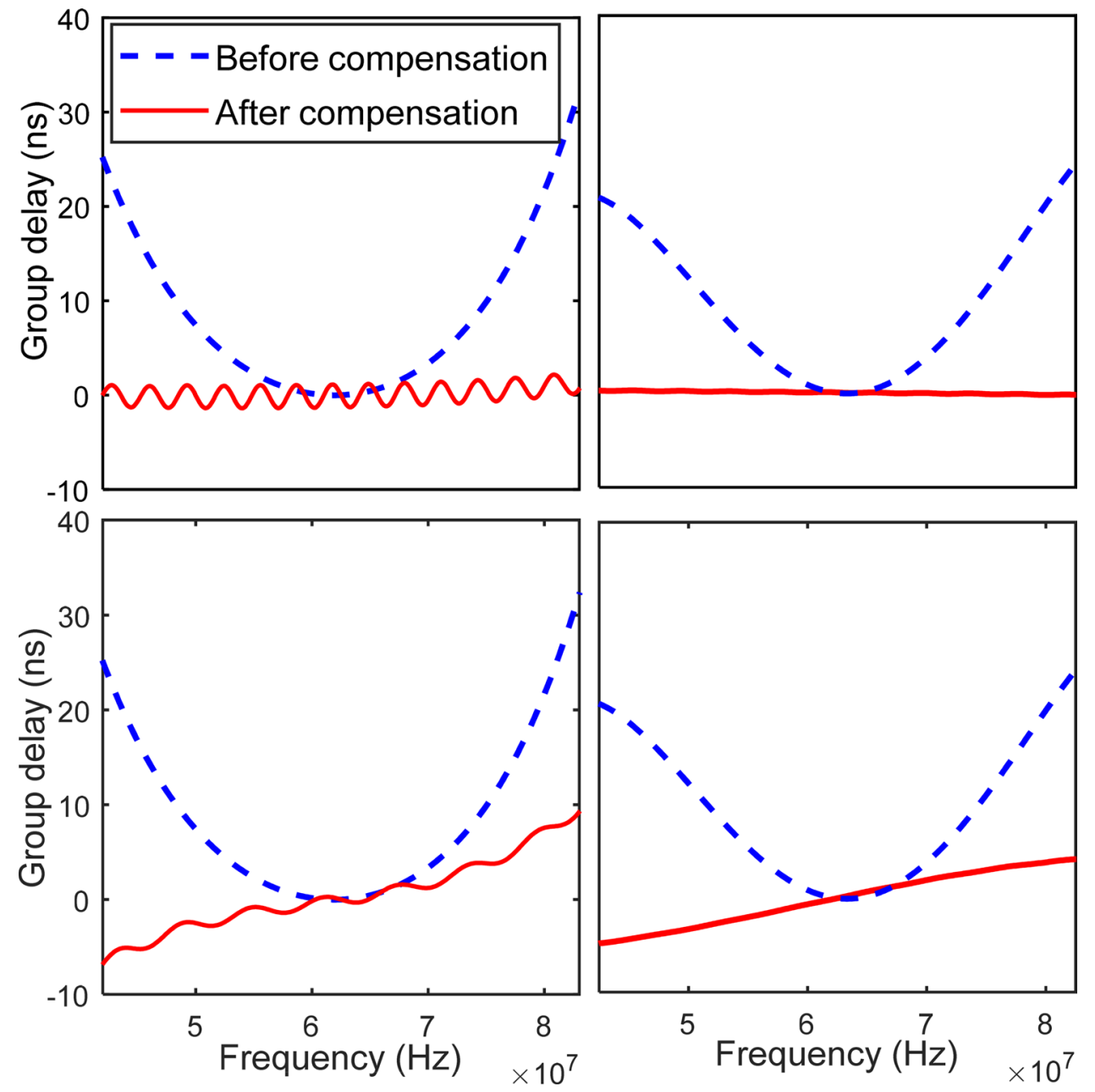
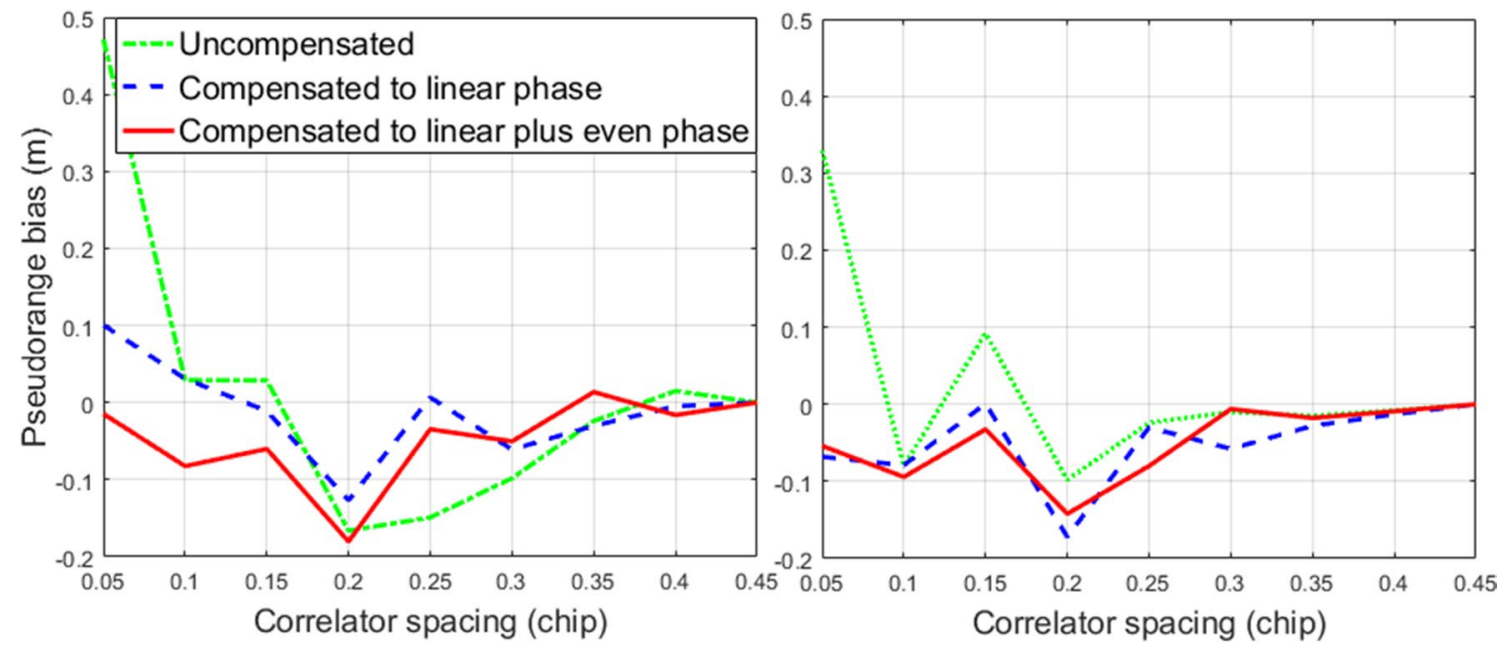

Fig. 14 Receiver-measured pseudorange biases before and after phase compensation for PRN32 (left) and PRN33 (right) satellite B1C channels 
significant; (3) the effects of receiver imperfections on pseudorange biases can aggravate or compensate the effects of satellite imperfections.

Second, a channel phase equalizer based on all-pass filter is constructed according to the linear phase criterion and the linear plus even-symmetric phase criterion, respectively. Its group delay fitting MSE and resulting SCB can be minimized by adjusting its order $N$ and parameter $\beta$. The simulation example of the BPSK(10) signal verifies the remarkable effect of the equalizer in reducing pseudorange biases and the similar effect and complexity of the two criteria. The two criteria as well as the target phase response of the second criterion can be selected flexibly according to the specific channel characteristics.

Finally, by using the B1C signals from the BDS3 PRN32 and PRN33 satellites collected by a high-gain parabolic antenna, the transfer function of the entire channel is inverted and a phase equalizer is constructed according to the above two criteria. The measurements of our software receiver show that the SCB is significantly reduced, and the effects of the two criteria are comparable.

Acknowledgements This study was supported by the National Natural Science Foundation of China (Grant No. 61271197).

Open Access This article is licensed under a Creative Commons Attribution 4.0 International License, which permits use, sharing, adaptation, distribution and reproduction in any medium or format, as long as you give appropriate credit to the original author(s) and the source, provide a link to the Creative Commons licence, and indicate if changes were made. The images or other third party material in this article are included in the article's Creative Commons licence, unless indicated otherwise in a credit line to the material. If material is not included in the article's Creative Commons licence and your intended use is not permitted by statutory regulation or exceeds the permitted use, you will need to obtain permission directly from the copyright holder. To view a copy of this licence, visit http://creativecommons.org/licenses/by/4.0/.

\section{References}

Abel JS, Smith JO (2006) Robust design of very high-order all-pass dispersion filters. In: Proceedings of the 9th international conference on digital audio effects (DAFx-06), Montreal, Canada, September 18-20, pp 13-18

Abel JS, Valimaki V, Smith JO (2010) Robust, efficient design of allpass filters for dispersive string sound synthesis. IEEE Signal Process Lett 17(4):406-409

Arikan F, Nayir H, Sezen U, Arikan O (2008) Estimation of single station interfrequency receiver bias using GPS-TEC. Radio Sci 43(4):1-13

Betz JW (2002) Effect of linear time-invariant distortions on RNSS code tracking accuracy. In: Proceedings of the ION GPS 2002, Institute of Navigation, Portland, OR, USA, September 24-27, pp 1636-1647

Guo N, Kou Y, Zhao Y, Yu Z, Chen Y (2014) An all-pass filter for compensation of ionospheric dispersion effects on wideband GNSS signals. GPS Solut 18(4):625-637
HaKansson M, Jensen ABO, Horemuz M, Hedling G (2017) Review of code and phase biases in multi-GNSS positioning. GPS Solut 21(3): $1-12$

He C, Lu X, Guo J, Su C, Wang W, Wang M (2020) Initial analysis for characterizing and mitigating the pseudorange biases of BeiDou navigation satellite system. Satell Navig 1(1):1-10

ICAO (2001) International standards and recommended practices, Annex 10, Attachment D

Liu T, Zhang B, Yuan Y, Li Z, Wang N (2019a) Multi-GNSS triplefrequency differential code bias (DCB) determination with precise point positioning (PPP). J Geod 93(5):765-784

Liu Y, Chen L, Yang Y, Pan H, Ran Y (2019b) Theoretical evaluation of group delay on pseudorange bias. GPS Solut. https://doi. org/10.1007/s10291-019-0861-z

Misra P, Enge P (2006) Global positioning system: signals, measurements, and performance, 2nd edn. Ganga-Jamuna Press, Lincoln, MA, pp 467-498

Montenbruck O et al (2017) The multi GNSS experiment (MEGX) of the international GNSS service (IGS) - achievements, prospects and challenges. Adv Space Res 59(7):1671-1697

Pagot JB, Julien O, Thevenon P, Fernandez FA, Cabantous M (2018) Signal quality monitoring for new GNSS signals. Navigation 65(1):83-97

Phelts RE (2001) Multicorrelator techniques for robust mitigation of threats to GPS signal quality. Dissertation, Stanford University

Phelts RE, Blanch J, Walter T, Enge P (2014) The effect of nominal signal deformation biases on ARAIM users. In: Proceedings of the ION ITM 2014, Institute of Navigation, San Diego, CA, USA, January $27-29$, pp 56-67

Proakis JG, Salehi M (2008) Digital communications, 5th edn. McGraw Hill, New York

Schaer S (2016) SINEX BIAS—solution (software/technique) INdependent EXchange Format for GNSS Biases Version 1.00. Dec 2016. http://ftp.aiub.unibe.ch/bcwg/format/draft/sinex_bias_100_ feb07.pdf. Accessed July 2020

Soellner M, Kohl R, Luetke W, Erhard P (2002) The impact of linear and nonlinear signal distortions on Galileo code tracking accuracy. In: Proceedings of the ION GPS 2002, Institute of Navigation, Portland, OR, USA, September 24-27, pp 270-1285

Soellner M, Kurzhals C, Hechenblaikner G, Rapisarda M, Burger T, Erker S, Furthner J, Grunert U, Meurer M, Tholert S (2008) GNSS offline signal quality assessment. In: Proceedings of the ION GNSS 2008, Institute of Navigation, Savannah, GA, USA, September 16-19, pp 909-920

Spilker JJ (1996) Fundamentals of signal tracking theory. In: Parkinson BW, Spilker JJ (eds) Global positioning system-theory and applications (Volume I). American Institute of Aeronautics and Astronautics, Washington DC, pp 245-327

Thöelert S, Vergara M, Sgammini M, Enneking C, Antreich F, Meurer M, Brocard D, Rodriguez C (2014) Characterization of nominal signal distortions and impact on receiver performance for GPS (IIF) L5 and Galileo (IOV) E1/E5a Signals. In: Proceedings of the ION GNSS+ 2014, Institute of Navigation, Tampa, FL, USA, September 8-12, pp 3113-3128

Van Dierendonck AJ (1996) GPS receivers. In: Parkinson BW, Spilker Jr. JJ (ed) Global Positioning System: theory and applications (Volume I). Progress in astronautics and aeronautics, Volume 163, American Institute of Aeronautics and Astronautics, Washington DC, ISBN 1-56347-106-X, pp 329-408

Vergara M, Sgammini M, Thoelert S, Enneking C, Zhu Y, Antreich F (2016) Tracking error modeling in presence of satellite imperfections. Navigation 63(1):3-13

Vergara M, Antreich F, Enneking C, Sgammini M, Seco-Granados $\mathrm{G}$ (2020) A model for assessing the impact of linear and nonlinear distortions on a GNSS receiver. GPS Solut. https://doi. org/10.1007/s10291-019-0917-0 
Wang N, Yuan Y, Li Z, Montenbruck O, Tan B (2016) Determination of differential code biases with multi-GNSS observations. J Geod 90(3):209-228

Wang N, Li Z, Duan B, Hugentobler U, Wang L (2020) GPS and GLONASS observable-specific code bias estimation: comparison of solutions from the IGS and MGEX networks. J Geod 94(8): $1-15$

Ward PW (2017) GNSS receivers. In: Kaplan ED, Hegarty CJ (eds) Understanding GPS/GNSS-principles and applications, 3rd edn. Artech House, Norwood, MA, pp 339-548

Yan T, Wang Y, Qu B, Liu X, Wang G (2018) Analysis methods of linear distortion characteristics for GNSS Signals. In: Proceedings of the China Satellite Navigation Conference (CSNC). Lecture notes in electrical engineering 499, Springer, Singapore, pp 183-195. https://doi.org/10.1007/978-981-13-0029-5_17

Zhu X, Li Y, Yong S, Zhuang Z (2008) A novel definition and measurement method of group delay and its application. IEEE Trans Instrum Meas 58(1):229-233

Publisher's Note Springer Nature remains neutral with regard to jurisdictional claims in published maps and institutional affiliations.

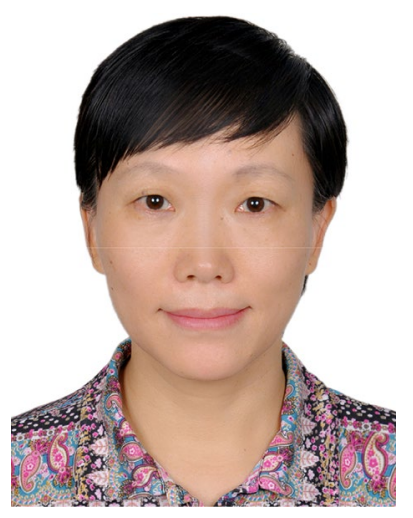

Yanhong Kou obtained a Ph.D. in Communication and Information System from Beihang University in 2006 and is an associate professor in the School of Electronics and Information Engineering at Beihang University, China. Her research interests include high-performance
GNSS receivers and simulators, signal processing, satellite communication. She is the author of $100+$ articles and has chaired $10+$ sessions for international conferences.

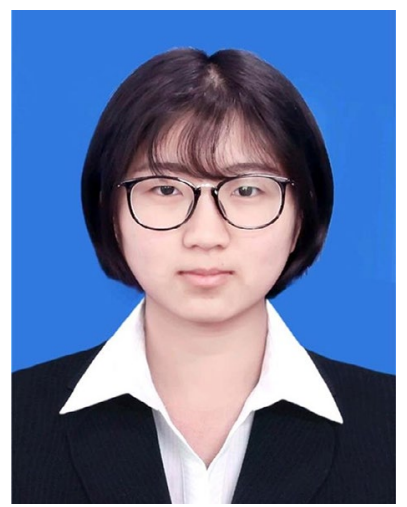

Huiwen $\mathrm{Wu}$ is a master's degree candidate in the School of Electronics and Information Engineering at Beihang University, China. She received her B.S. degree in information engineering from Nanjing University of Aeronautics and Astronautics in 2018. Her research focuses on GNSS signal processing and channel optimization. 